\title{
Mental Images Of Suicide: Theoretical Framework And Preliminary Findings In Youth
}

De Rozario $M R^{\star 1,2}$, Van Velzen $\mathrm{LS}^{\star 1,2}$, Davies $\mathrm{P}^{1,2}$, Rice $\mathrm{SM}^{1,2}$, Davey $\mathrm{CG}^{3}$, Robinson $\mathrm{J}^{1,2}$, Alvarez-Jimenez $\mathrm{M}^{1,2}$, Allott $\mathrm{K}^{1,2}$, McKechnie $\mathrm{B}^{1}$, Felmingham $\mathrm{KL}^{4}$, Schmaal $\mathrm{L}^{1,2}$

${ }^{*}$ Authors share first authorship

${ }^{1}$ Orygen, Parkville, Australia

${ }^{2}$ Centre for Youth Mental Health, University of Melbourne, Melbourne, Australia

${ }^{3}$ Department of Psychiatry, University of Melbourne, Melbourne, Australia

${ }^{4}$ Department of Psychological Medicine, University of Melbourne, Melbourne, Australia

\section{Corresponding author:}

Name: $\quad$ Lianne Schmaal

Affiliation: Orygen

Centre for Youth Mental Health, University of Melbourne

Address: $\quad 35$ Poplar Road, Parkville, VIC 3052, Australia

Telephone: +61399669524

Email: $\quad$ lianne.schmaal@unimelb.edu.au 


\section{ABSTRACT}

Suicide is the second leading cause of death in young people between 10 and 24 years old. There is a clear need to identify novel treatment targets in order to develop more effective strategies to reduce the massive impact of youth suicide. This paper discusses 'suicidal flashforwards' as a potential novel target. Suicidal flashforwards are vivid mental images of future death or suicide - a form of suicidal cognition that has received very little attention in both research and clinical practice to date. Here we present a brief review of relevant literature and propose potential clinical and cognitive factors associated with suicidal flashforwards, and the progression from suicidal flashforwards to suicidal behaviours (e.g., suicide attempt). In addition, we present data from our pilot study on suicidal flashforwards, which is the first study to examine this phenomenon in young people. Thirty-three young people (aged 16-25) with current or recent (in past 4 weeks) suicidal ideation, who received treatment at a local youth mental health clinic for a mood disorder participated in the study. Our findings suggest that suicidal flashforwards are very common in this population (reported by $97 \%$ of the sample), and are experienced as vivid, real, distressing and intrusive. Importantly, we found an association between higher ratings of distressing effects of suicidal flashforwards and the number of previous suicide attempts, while no associations with attempts were found for characteristics of verbal suicidal thoughts. These findings highlight the importance of suicidal flashforwards as a potential target for risk management and treatment strategies for suicide. Future larger studies that aim to understand the clinical, cognitive and biological mechanisms underlying suicidal flashforwards are needed to identify targets for appropriate preventions and interventions.

Keywords: suicide, mental imagery, youth, adolescents, suicidal flashforwards

Number of words: 4397

Number of Figures: 4 


\section{INTRODUCTION}

Suicide is a leading cause of death worldwide, with one death by suicide occurring every 40 seconds, and an estimate of one million people taking their own lives every year [1]. This important public health concern is even more pressing in young people, as suicide is the second leading cause of death in young people between 10 and 24 years old [1]. Despite national and international prevention strategies to reduce suicidal deaths, the suicide rates in most countries have shown a rising trend in recent years [2].

Unfortunately, no gold-standard, highly effective treatments for suicidal thoughts currently exist. Systematic reviews and meta-analyses have shown that interventions targeting suicidal thoughts and behaviours have limited efficacy and many of the treatments that do show some potential promise have evidence from only a single randomised controlled trial $[3,4]$. This is especially true in young people; the population at highest risk for suicide [5, 6]. Moreover, our understanding and ability to identify predictors of suicide or suicide attempts is also limited. A recent meta-analysis by Franklin et al. [7], summarised 50 years of research on predictors of suicide. Their results indicated that there have been no significant improvements over the years in our ability to predict who is likely to attempt or die by suicide, and that we are still operating at chance levels in our attempts to do so, which hampers suicide prevention efforts.

Given the gravity of outcomes surrounding suicide, there is an urgent need to identify novel treatment targets and mechanisms of suicidal ideation and suicide attempt, in order to develop better-informed and more effective treatment and risk management strategies. Suicide research and suicide risk assessment in clinical practice mainly focus on verbal thoughts of suicide. Here, we focus on a type of suicidal cognition that has received little attention to date: mental images of suicide. In this review, we will describe mental images of suicide, suggest potential clinical and cognitive correlates and discuss why some of these cognitive factors could be involved in 
mediating the transition from suicidal mental images to suicidal behaviours (including suicide attempt). In addition, we will present findings from our pilot study, which was the first to examine characteristics of mental images of suicide specifically in young people (aged 16-25).

\section{SUICIDAL FLASHFORWARDS}

Humans rely on at least two modes of thought: verbal (inner speech) and visual (imagery). Although past research efforts and clinical treatment of suicidal ideation have generally focused on verbal expressions of suicidal thoughts, it is increasingly recognised that many people with suicidal ideation also experience visual images of suicide. These visual images of suicide are described as realistic, compelling, and detailed mental images of being dead or of a future suicide attempt. Because these mental images of future death or suicide seem to mimic the powerful, intrusive and repetitive nature of the past-trauma flashbacks that are common in posttraumatic stress disorder, but instead are future-oriented, they have also been referred to as 'suicidal flashforwards' [8].

Research on mental imagery in general indicates that prospective imagery (i.e., imagery of future events) enables people to simulate and pre-experience the future and make decisions [9]. It has been proposed that suicidal flashforwards may potentially be more distressing and realistic than verbal thoughts of suicide. Studies have shown that future events that have been imagined are rated as more probable than those that have not been imagined, possibly because mental imagery enables individuals to identify barriers to realising the event [10-13]. In addition, mental images are both more emotionally arousing and more likely to be confused with real events than verbal thoughts of the same content [14]. The mental simulation of future events can provide great adaptive value (e.g., for athletes), as it supports planning, problem solving, and carrying out prospective intentions [15]. However, when applied to the context of suicidal ideation, it could have devastating consequences. 
Holmes and colleagues [8] were the first to describe suicidal flashforwards. In their study in 15 adults, every participant who had a history of both depression and suicidal ideation reported having experienced these intrusive flashforwards of suicide during their most vulnerable periods. Since Holmes and colleagues' seminal study, a few other studies have supported the notion that experiencing vivid, realistic and detailed mental images related to death and suicide are very common in adults with a mood disorder (both unipolar and bipolar depressive disorders) and suicidal ideation: across these studies $37 \%$ (in an epidemiological sample) to $100 \%$ (in clinical samples) reported experiencing suicidal flashforwards [16-18]. Importantly, individuals with suicidal flashforwards had more severe suicidal ideation than individuals without suicidal flashforwards [18] and reported a greater preoccupation with suicidal flashforwards than with suicide-related verbal thoughts [17].

Due to the high prevalence of suicidal flashforwards in adults with suicidal ideation and mood disorders, and the potential role of mental imagery in facilitating suicidal behaviour, suicidal flashfowards may represent an important target for suicide prevention and novel interventions. However, in order to develop appropriate risk management strategies and treatments for suicidal flashforwards, it is important to understand their clinical and cognitive correlates and identify potential mechanisms by which suicidal flashforwards could facilitate suicidal behaviour. Below, we discuss potential clinical and cognitive mechanisms associated with suicidal flashforwards. Because the (very limited) evidence on suicidal flashforwards and their correlates that is available to date is based on research in suicidal adults, it is important to elucidate whether suicidal flashforwards are also common in young people. Therefore, we will additionally discuss novel findings from our pilot study examining suicidal flashforwards and their clinical correlates in young people. 


\section{CLINICAL AND COGNITIVE CORRELATES OF SUICIDAL FLASHFORWARDS}

Previous studies have found associations between mood disorders, suicidal flashforwards, and the overall severity/intensity of suicidal ideation. We propose that a number of clinical, behavioural and cognitive factors may be influenced by, as well as influence one's vulnerability for suicidal flashforwards. While we have tried to address a range of potential correlates (Figure 1), we acknowledge that this is not an exhaustive overview.

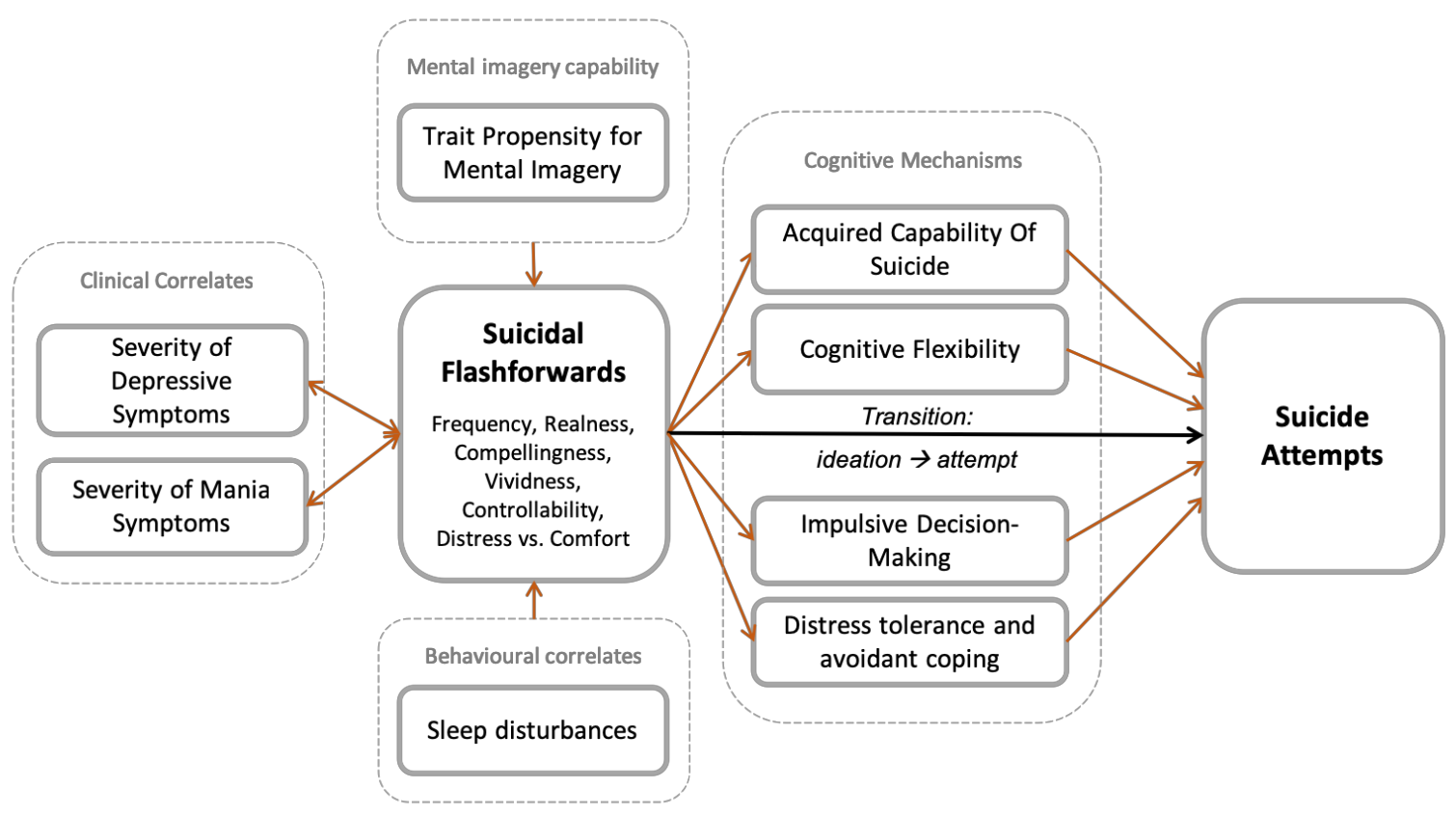

Figure 1: Potential clinical, behavioural and cognitive correlates of suicidal flashforwards.

\section{Severity of Mood Disorder Symptoms}

One large web-based study examined associations between occurrence of mental imagery (negative, positive and suicide-related imagery) and severity of depressive symptomatology in a sample of over nine hundred participants with various self-reported psychiatric disorders (e.g. depression, bipolar disorder, addiction, borderline personality disorder, social anxiety disorder and/or PTSD) [19]. Higher frequency of negative and suicide-related imagery and lower occurrence of positive imagery was related to greater severity of depressive symptoms at 
baseline and at follow-up. Interestingly, the images related to self-injury and death were mostly described as pleasant, which may be related to the high controllability and low intrusive nature of these images in this sample. The finding that negative and suicide- and death-related mental imagery were most common in those with most severe depressive symptoms are in line with the postulation that mental imagery may exacerbate or amplify mood states and affect mood stability in patients with mood disorders $[20,21]$. This is further supported by findings of an experimental study showing that the number of hypomanic-like experiences was associated with greater mood amplification after a positive imagery task [22]. Nonetheless, it remains unclear whether suicidal flashforwards are limited to or more common in individuals with more severe mood disorder symptoms, or whether the experience of suicidal mental images increases the severity of mood disorder symptoms.

\section{Trait Propensity for Mental Imagery}

There are strong inter-individual differences in visual imagery ability (e.g. generation of mental images, vividness and control over mental images) [23, 24]. Additionally, the degree to which an individual uses mental imagery in daily life also varies between individuals. This can be assessed using the Spontaneous Use of Imagery Scale (SUIS) [25]. Scores on the SUIS were found to be significantly correlated with the vividness of mental imagery, with individuals that experience vivid mental images reporting higher use of imagery in everyday life [25]. These inter-individual differences appear to link to psychopathology in interesting ways. In one study, Morina and colleagues [26] showed participants videos of distressing events and found that the vividness of general mental imagery prior to viewing the images was positively associated with the occurrence of intrusive imaginal memories following the stressful event, suggesting that greater mental imagery abilities may be a risk factor for intrusive trauma-related mental images. 
Specifically related to suicidal flashforwards, studies by Hales et al. [17] and $\mathrm{Ng}$ et al. [18] suggest that being a visualiser and having a greater overall tendency to generate mental images is associated with the impact and compellingness of suicidal flashforwards and potentially with risk for suicide. Using the SUIS, Hales et al. [17] showed that individuals with bipolar disorder who considered suicide-related flashforwards as more compelling than individuals with major depressive disorder, also had a higher trait propensity for mental imagery than those with major depressive disorder. While evidence is still limited regarding the association between trait propensity for mental imagery and suicidal flashforwards, we propose that individual differences in trait propensity for mental imagery may be a predisposing factor for suicidal flashforwards (Figure 1).

\section{Sleep Disturbances}

Research suggests that sleep quality has the propensity to modulate an individual's capacity to actively generate and exert control over mental images. Sleep quality has been linked to motor imagery learning $[27,28]$ and mental imagery ability in athletes [29]. Good sleep quality has been found to have protective effects on the development of subsequent intrusive memories and images. For example, studies have found that the number of intrusive memories/images following exposure to traumatic stimuli is reduced after sleep [30, 31]. Conversely, poor sleep quality has been found to exacerbate symptoms in PTSD patients, including intrusive memories and nightmares [30]. Recent evidence suggests that sleep disturbances in the form of too little sleep or too much sleep following a traumatic event posed a risk for developing trauma-related intrusive mental images in the following week [32]. Given the similarities between intrusive memories and images (i.e., flashbacks) in PTSD and suicidal flashforwards, we speculate that insomnia and sleep disturbances may exacerbate suicidal flashforwards, potentially through 
impairment of cognitive control [33, 34], thereby increasing the intrusiveness of suicidal flashforwards.

Sleep is also known to play an important role in emotion-regulation. Sleep disturbances have been associated with an increase in emotional reactivity [35], and have been shown to increase distress associated with trauma-related mental images [36]. Similarly, sleep disturbances may increase the emotional impact of suicidal flashforwards, including their distressing effects, although this remains to be investigated.

\section{Acquired Capability of Suicide}

In addition to clinical correlates of suicidal flashforwards, additional cognitive mechanisms may be involved in the maintenance of suicidal flashforwards and may mediate the transition from suicidal flashforwards to suicide attempt (Figure 1). One such mechanism may involve an acquired capability of suicide. The notion of an acquired capability of suicide was proposed as part of Joiner's Interpersonal-Psychological Theory of Suicide [37]. According to this theory, there are three contributing factors that explain suicidal behaviour: (1) perceived burdensomeness, (2) thwarted belongingness (e.g., loneliness and the absence of reciprocal care), and (3) capability of suicide. The theory posits that suicidal desire emerges when individuals experience intractable feelings of perceived burdensomeness and thwarted belongingness, and that near-lethal or lethal suicidal behaviour occurs in the presence of both suicidal desire and capability for suicide. Hence, according to this theoretical model, an acquired capability for suicide is a necessary precursor for suicidal behaviours.

Capability for suicide develops after repeated exposures to painful and provocative events (e.g., self-injurious behaviour), which in turn lowers one's fear of death and elevates one's physical 
pain tolerance, perhaps through habituation to such experiences. The theory posits that, with the accumulation of exposure to such experiences, an individual progressively adopts a higher tolerance of pain, as well as a fearlessness of death. Acquired capability for suicide may be particularly relevant in the context of suicidal flashforwards, because the accumulated, repeated experience of visually imagining one's own future suicide attempt or death could act in the same way as rehearsing or practicing other behaviours that may lower one's fear of death and increases ones pain tolerance [38, 39]. From this perspective, suicidal flashforwards could be conceptualised as a means of habituation, inhibiting the fear response towards hurting oneself and the pain associated with suicide. Thus, we hypothesise that with increased mental imagination of a prospective suicide attempt or death, a decrease in fear of death and of the pain associated with suicide occurs, which may lead to an elevated risk of engaging in suicidal behaviour. Although increasing evidence supports the role of acquired capability of death in the transition from ideation to attempt [40], the links between acquired capability of death, suicidal flashforwards and the risk of acting on suicidal flashforwards remains to be investigated.

\section{Cognitive Rigidity}

In addition to acquired capability of suicide, reduced cognitive flexibility may be a potential mechanism that mediates the transition from suicidal flashforwards to suicide attempt (Figure 1). Cognitive flexibility is defined as the capability of an individual to respond to environmental change in an adaptive manner [41]. Poor cognitive flexibility limits one's ability to generate alternative solutions to a problem, causing one to fixate on automatic, less adaptive responses to the given situation. The generation of suicidal flashforwards could increase the cognitive availability of suicidal behaviour [42]. As an individual with suicidal thoughts goes through any life crisis, they may react to the crises with the solutions most cognitively available to them, especially if they lack the cognitive flexibility to think of alternative solutions or alternative means to cope. Cognitive inflexibility/rigidity may inflate the chance that any crisis experienced by 
individuals who experience suicidal flashforwards will turn into a suicidal crisis. Various studies have supported the association between cognitive rigidity and suicidal cognitions and behaviour in general $[43,44]$. However, associations between cognitive flexibility and suicidal flashforwards in specific remain to be elucidated.

\section{Impulsivity}

Impulsivity may be the cognitive construct that has received the most research interest in suicidal populations [45]. Many studies support a link between impulsivity and suicidal ideation/attempt $[46,47]$. While there are multiple components that comprise the phenomenon of impulsivity, we postulate that impulsive decision making is one aspect of impulsivity that may be of particular relevance to suicidal flashforwards.

Impulsive decision making is defined as the act of making decisions that favour immediate or short-term gratification over larger, delayed rewards [48]. Delay, by nature, devalues rewards; however, the tendency to devalue rewards based on the extent of the delay (known as temporal discounting) [49] can be offset when individuals believe a reward is of great enough (emotional) value to them [50]. The ability to delay rewards in order to gain more valuable rewards over time differs across people; some individuals have a greater tolerance for delay than others. This tendency can be tested using delay discounting tasks that identify the point at which an individual is willing to forgo a larger delayed reward for a smaller, immediate one [51]. The longer a person is willing to wait for a larger but delayed reward compared to a smaller immediate reward, the less impulsive the person is considered to be. Hypothetical monetary rewards are typically used in delay discounting tasks; however, the underlying mechanisms of delay tolerance and impulsive decision tendencies can apply to any kind of reward, including suicidal behaviours or suicide death if regarded as a desirable outcome. 
Vivid and detailed visual images of future death or suicide can be perceived as comforting and as a desirable outcome, by representing routes to escaping the distress one experiences. For an individual who is prone to impulsive decision making, the immediacy of escape from distress can be viewed as reinforcing and desirable - potentially overshadowing the possibility that alternative solutions will lead to a more optimal resolution to the crisis over the longer term. The presentation of an immediate solution to distress via suicidal flashforwards may trigger an impulse decision to act in accordance with the images. Given these potential mechanisms, we propose that the higher the tendency of a person to make impulsive decisions, the more likely they are to act on suicidal flashforwards and engage in suicide attempt (Figure 1). It is worth noting that this hypothesis may be specific to explaining a relationship between suicidal flashforwards and impulsive suicide attempts, and in particular in people who regard suicidal flashforwards as comforting and rewarding (e.g., by representing a means to escape distressing emotions and life circumstances), as opposed to more carefully planned suicides and in people who experience these images as distressing.

\section{Distress Intolerance and Escape/Avoidant Coping}

The way people cope or react to suicidal flashforwards may determine how frequent, distressing and intrusive they are. For example, if suicidal flashforwards are experienced as intrusive and distressing, they may exacerbate negative emotional states, especially in individuals with low distress tolerance. In accordance with the Escape Theory of Suicide [52], suicide or suicidal behaviours can be perceived as a desirable outcome, by representing routes to escape from the distress or the emotional pain associated with suicidal flashforwards. Therefore, we hypothesise that individuals with lower distress tolerance may be more inclined to engage in suicidal behaviours and to transition from suicidal flashforwards to suicide attempt (Figure 1). Maladaptive coping styles such as escape/avoidance may also intensify the very experience that an individual is trying to escape [53-55]. Therefore, we hypothesise that an 
escape/avoidant coping style in response to suicidal flashforwards will increase the frequency, vividness and intrusiveness of subsequent suicidal flashforwards, thereby in turn potentially mediating the transition to suicidal behaviours (Figure 1).

\section{SUICIDAL FLASHFORWARDS IN YOUNG PEOPLE: A PILOT STUDY}

Given that our (still limited) knowledge on suicidal flashforwards is based on research conducted in adults with suicidal ideation (mean age of referenced studies, $M=40.66$ years), we conducted a pilot study to determine whether suicidal flashforwards are also commonly experienced by young people; the population with highest suicide risk. We examined the characteristics of suicidal flashforwards in 33 young people (aged 16-25) who received treatment for a mood disorder (unipolar or bipolar depressive disorder) at a youth mental health clinic in Melbourne and who reported recent (in past 4 weeks) suicidal ideation. For reasons of ensuring the safety of the young people participating in the study, young people (1) at imminent risk for suicide (i.e., individuals with current suicidal ideation with a plan, as well as the means and intent to enact on the plan), and (2) who were actively manic or psychotic, were excluded from participation in the study.

The content and characteristics of suicidal flashforwards were assessed with the Suicidal Flashforwards and Cognitions Interview (SCFI) [8]. The SCFI is a structured interview assessing the content of visual imagery of suicide as well as verbal suicidal thoughts at the time participants felt at their most suicidal, and have them rate (1) how much time they spent preoccupied with suicide-related imagery and verbal thoughts, (2) how compelling and real these images and verbal thoughts were, (3) how controllable they were, (4) how distressing and comforting they were; (5) how vivid the images were; (6) how intense the urge to act on these images/thoughts were. Additional questions in the SCFI included whether the images were observed from a field or observer perspective and what the images mean to the participants. 
Participants were asked to describe their most significant suicidal flashforward in as much detail as possible, including the scenes, actions, locations, people present, other sensory experiences (smell, taste, sound, touch) and feelings. In order to investigate relationships with the potential clinical correlates proposed in Figure 1, the young people were also asked to respond to a number of self-report measures. These measures included the Quick Inventory of Depressive Symptomatology (QIDS) [56], Altman Self-Rating Mania Scale (ASRM) [57], Pittsburgh Sleep Quality Index (PSQI) [58], Spontaneous Use of Imagery Scale (SUIS) [25] and ColumbiaSuicide Severity Rating Scale (C-SSRS) [59].

We found that suicidal flashforwards are very common in (if not almost unanimously experienced by) young people with present suicidal ideation and a mood disorder. Of the 33 participants in the sample, $32(97 \%)$ reported experiencing suicidal flashforwards - with the majority of the sample reporting that they experienced flashforwards often $(n=21 ; 64 \%)$. Of the 32 who experienced suicidal flashforwards, 18 (56\%) reported that mental images of their future suicide attempt or self-harm constituted the main content of any mental imagery they experienced (Figure 2A). Another prominent theme was that of the impact of the young person's death on others. Importantly, $83 \%$ of the young people whose suicidal flashforwards primarily constituted envisioning their own future death or suicide, indicated that the images increased their urge to act on these images (Figure 2B). 


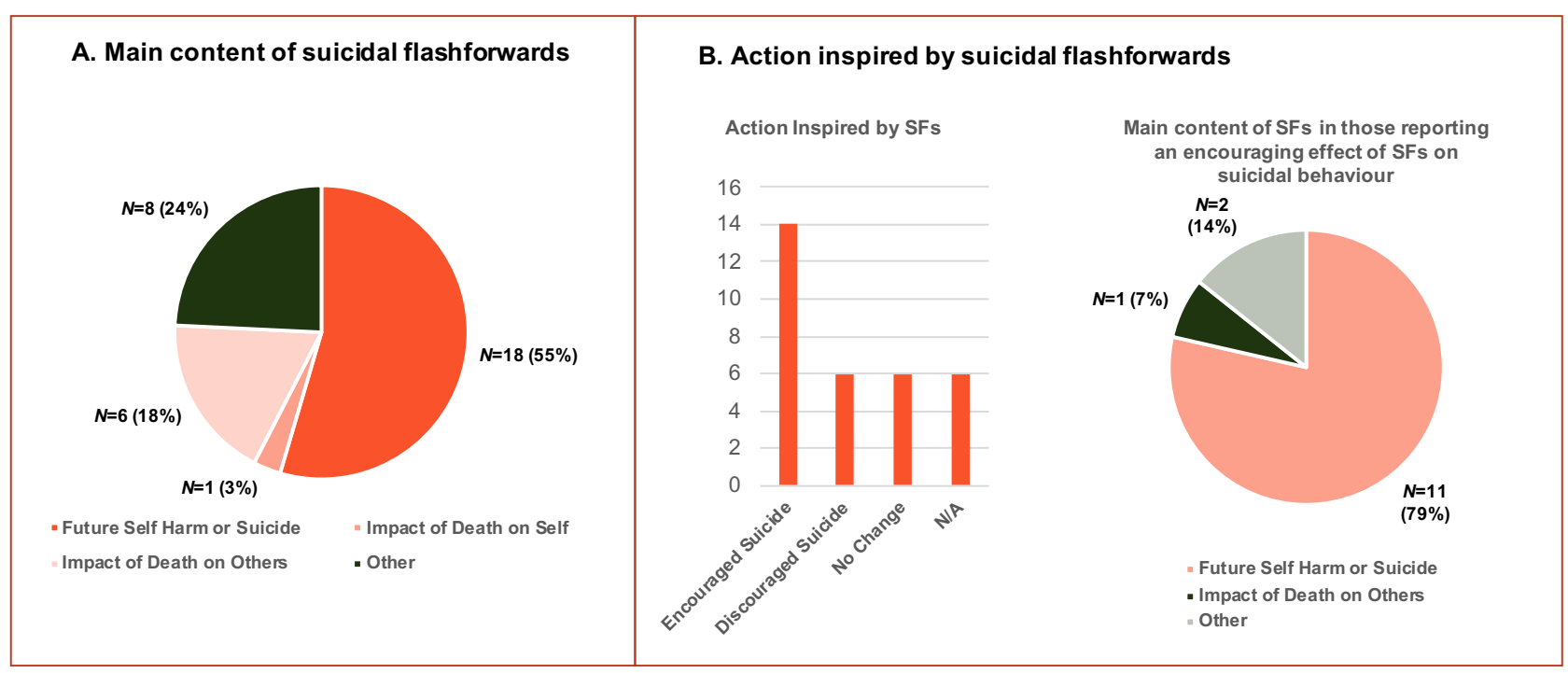

Figure 2. (A) Main content of suicidal flashforwards in our sample of young people with suicidal flashforwards; (B) Action inspired by suicidal flashforwards. SFs: suicidal flashforwards.

The majority of the young people who experienced suicidal flashforwards reported their imagery of suicide to be realistic, compelling, and vivid (Figure 3). While previous studies in adults found that suicidal flashforwards can be both comforting and distressing $[8,16]$, the majority of the young people in our pilot rated them as distressing, with very few young people rating the suicidal flashforwards as comforting (Figure 3). In addition, most young people indicated that they did not have control over the suicidal flashforwards, indicating that the images are mostly experienced as intrusive in this sample (Figure 3). 

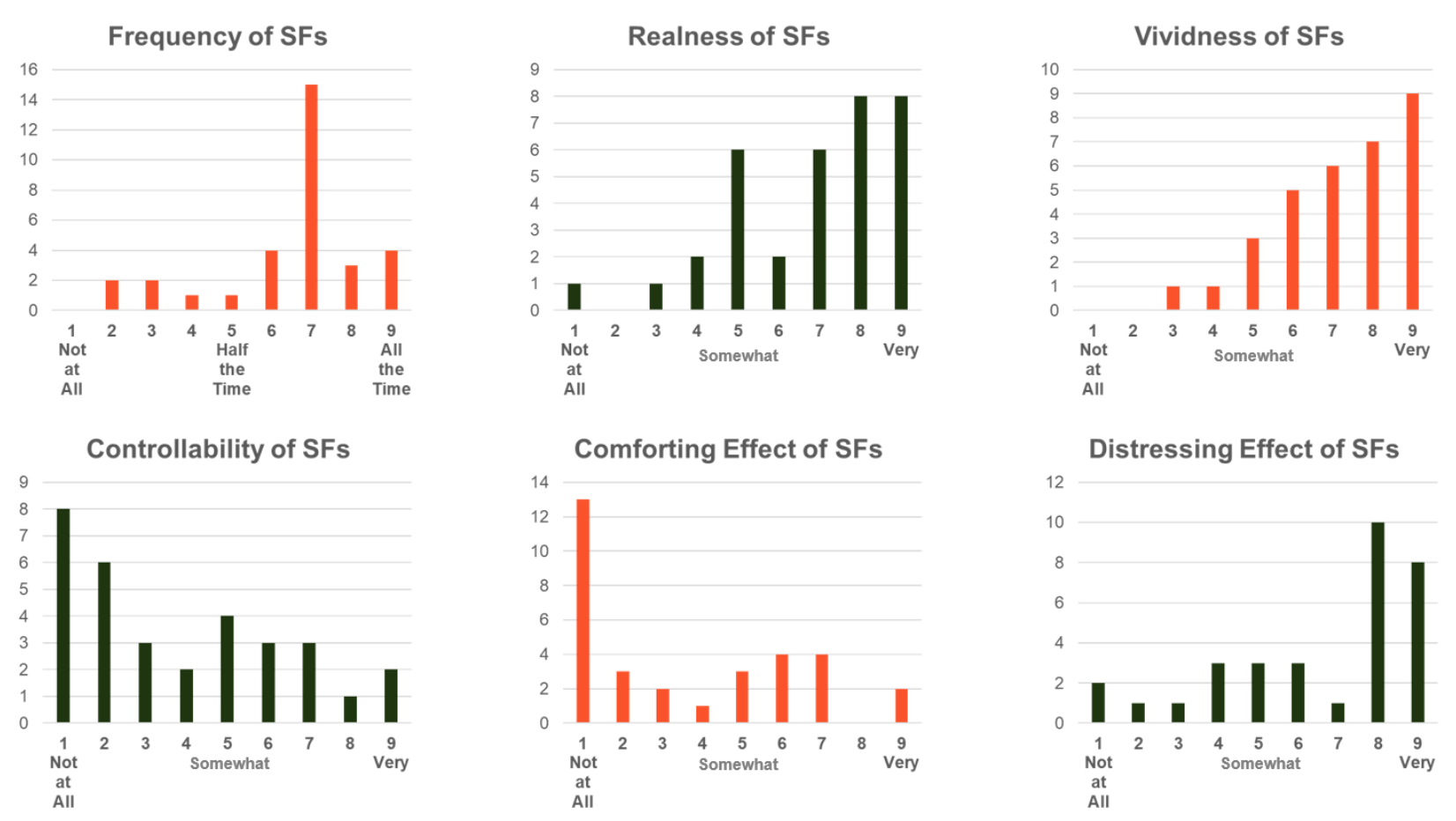

Figure 3. Ratings of characteristics of suicidal flashforwards in $\mathrm{N}=32$ young people with suicidal flashforwards. SFs: suicidal flashforwards.

In our pilot study, 25 of the young people had a history of at least one suicide attempt. Importantly, we found that ratings of distress associated with suicidal flashforwards were positively associated with suicidal behaviour (i.e., the cumulative total of past actual, aborted and interrupted attempts; $r=0.42, p=0.016$ ), while no association between any of the characteristics of verbal suicidal thoughts and number of attempts was found.

With regard to clinical characteristics, we found significant correlations between greater overall sleep disturbances, as measured with the PSQI total score, and higher ratings of vividness $(r=$ $0.54, p=0.016)$ and realness $(r=0.44, p=0.017)$ of the suicidal flashforwards (Figure 4). Higher ratings of the realness of the suicidal flashforwards were also associated with a higher trait propensity for mental imagery (SUIS total score; $r=0.41, p=0.021$ ) and greater depressive symptoms severity (QIDS total score; $r=0.39, p=0.029$ ) (Figure 4). Greater severity of manic 
symptoms (ASRM total score) was negatively correlated with the controllability of the suicidal flashforwards $(r=-0.39, p=0.029)$.
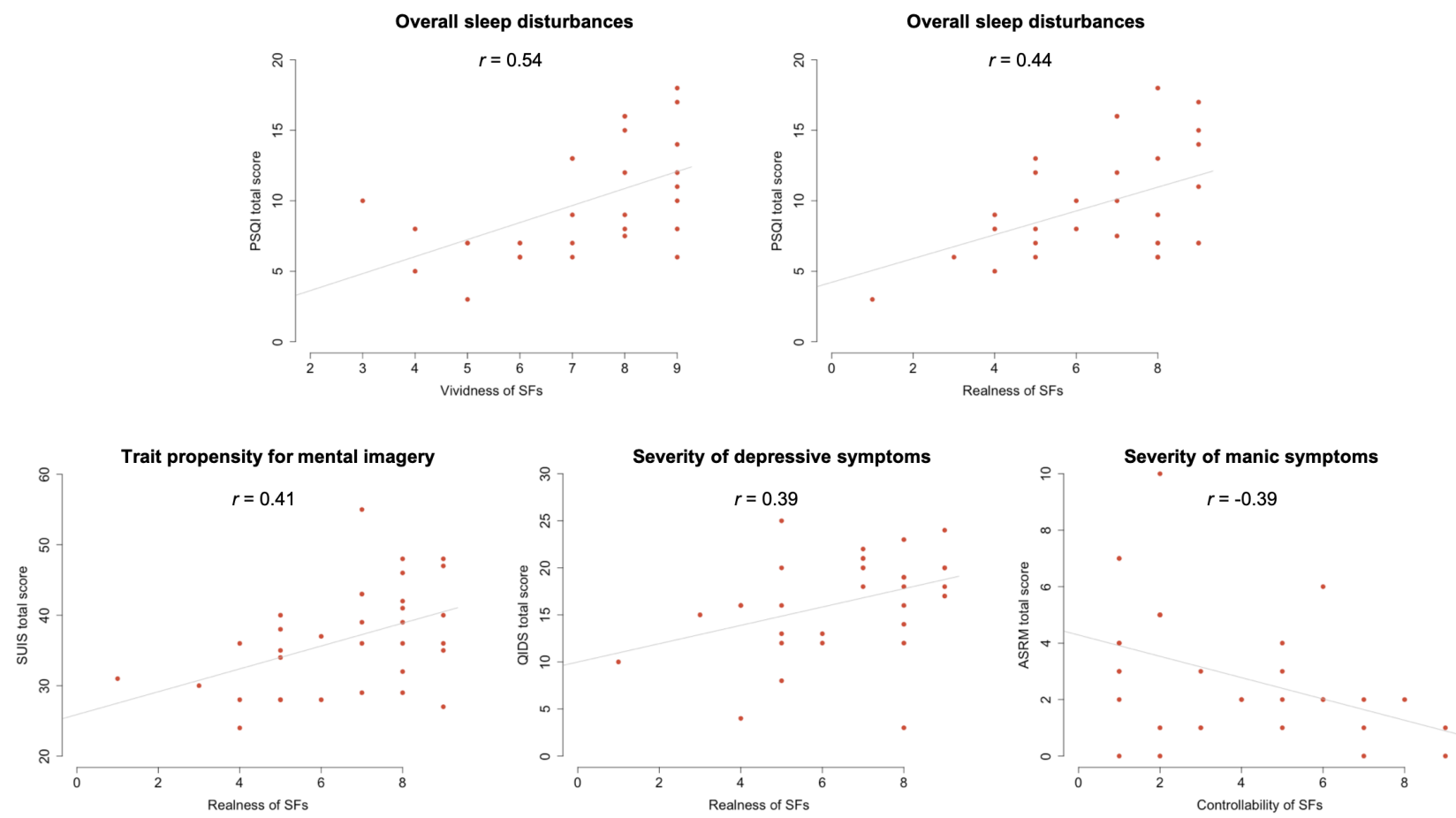

Figure 4. Scatterplots of associations between clinical and cognitive correlates and characteristics of suicidal flashforwards.

\section{CONCLUSIONS AND FUTURE DIRECTIONS}

The literature reviewed and initial findings presented indicate that suicidal flashforwards are a form of suicidal cognitions that is highly prevalent across age groups. Previous findings and findings from our pilot study show links between characteristics of suicidal flashforwards and greater severity of depressive and manic symptoms in people with a mood disorder. Our preliminary findings in young people also highlight a potential role of sleep disturbances in the vividness and realness of suicidal flashforwards, suggesting that disturbed sleep may be an indirect target for treatment of suicidal flashforwards. Importantly, in line with a previous study in 
adults [60], our preliminary findings in youths show that suicidal flashforwards, but not verbal thoughts of suicide, differentiate between those who ideated about suicide but not attempted suicide and those who attempted suicide. These findings support our hypothesis that suicidal flashforwards, more than verbal thoughts of suicide, are important for the progression from suicidal ideation to suicidal behaviours. Although these findings are preliminary and will need to be confirmed in larger samples, together with observations that suicidal flashforwards predict an increase of suicidal ideation over time [18], they underscore the importance of targeting suicidal flashforwards in suicide risk management and treatment. Unfortunately, clinical practice as well as suicide research have mainly focused on suicidal ideation in verbal form and seldom explore the presence of suicidal mental images. For example, Hales et al. [17] found that less than a quarter of the 40 participants in their study had discussed their suicidal images with their counsellor. This is in line with our pilot study, in which both the young people and their treating clinicians indicated that suicidal images were hardly ever discussed in treatment.

Longitudinal studies are required to evaluate whether experiencing suicidal flashforwards, or specific characteristics of suicidal flashforwards, constitute a risk factor for transitioning to suicide attempt. Such longitudinal studies would benefit from including measures of mechanisms that may mediate the progression from suicidal flashforwards to suicide attempt. In the current paper, we have proposed various cognitive mechanisms that could increase the risk of acting on the suicidal flashforwards (e.g., acting in accordance to the content of the visual images of future suicide), including acquired capability of suicide, cognitive rigidity, impulsive decision making, distress intolerance and an avoidant coping style. An investigation of the associations between these suggested cognitive mechanisms and suicidal flashforwards in young people is currently underway. 
Treatment strategies for suicidal flashforwards that have been proposed, include (but are not limited to): imaginal exposure, imagery rescripting, systematic desensitisation, eye movement desensitisation and reprocessing (EMDR), imagery-focused cognitive behaviour therapy (CBT), and positive imagery training $[38,61-64]$. These interventions may represent stand-alone treatments or additions to traditional psychological interventions for a distressing and underrecognised aspect of suicidal ideation. Given that these imagery-based therapies target distinct aspects of disturbing mental imagery, a better understanding of the cognitive and neurobiological mechanisms involved in suicidal flashforwards could help decide which intervention should be prioritised. For example, identifying the neural mechanisms underlying the generation and maintenance of suicidal flashforwards could provide important information on how to potentially disrupt suicidal flashforwards and may stimulate further treatment-oriented research via the identification of novel neural mechanisms. To date, no studies have directly examined the neural mechanisms underlying suicidal flashforwards. This type of research would increase our understanding of the underlying pathophysiology, may reveal novel treatment targets, and may provide biomarkers for monitoring the effects of treatment. For example, transcranial magnetic stimulation of regions that modulate the intrusiveness of suicidal mental images could help boost control over these images. Better control over mental imagery of negative emotional events have been shown to reduce involuntary intrusive visual images and reduce feelings of worry about feared scenarios $[65,66]$. In addition, neurofeedback could enable people to learn to regulate patterns of local brain activation that modulate the intrusiveness, vividness and emotional impact of suicidal flashforwards, through use of real-time feedback of these brain signals. By providing neurofeedback, individuals are enabled to adapt their cognitive strategies (e.g., for self-regulating distress during suicidal imagery) based on whether the strategies successfully contribute to achieving the desired brain target state. 


\section{REFERENCES}

1. World Health Organization. Preventing suicide: A global imperative. World Health Organization; 2014.

2. Alicandro G, Malvezzi M, Gallus S, La Vecchia C, Negri E, Bertuccio P. Worldwide trends in suicide mortality from 1990 to 2015 with a focus on the global recession time frame. Int J Public Health. 2019;64:785-795.

3. Glenn CR, Franklin JC, Nock MK. Evidence-based psychosocial treatments for selfinjurious thoughts and behaviors in youth. J Clin Child Adolesc Psychol. 2015;44:1-29.

4. Riblet NBV, Shiner B, Young-Xu Y, Watts BV. Strategies to prevent death by suicide: metaanalysis of randomised controlled trials. Br J Psychiatry. 2017;210:396-402.

5. Busby DR, Hatkevich C, McGuire TC, King CA. Evidence-Based Interventions for Youth Suicide Risk. Curr Psychiatry Rep. 2020;22:5.

6. Robinson J, Bailey E, Witt K, Stefanac N, Milner A, Currier D, et al. What Works in Youth Suicide Prevention? A Systematic Review and Meta-Analysis. EClinicalMedicine. 2018;45:52-91.

7. Franklin JC, Ribeiro JD, Fox KR, Bentley KH, Kleiman EM, Huang X, et al. Risk factors for suicidal thoughts and behaviors: A meta-analysis of 50 years of research. Psychol Bull. 2017;143:187-232.

8. Holmes EA, Crane C, Fennell MJV, Williams JMG. Imagery about suicide in depression-'Flash-forwards'? J Behav Ther Exp Psychiatry. 2007;38:423-434.

9. Schacter DL, Addis DR, Hassabis D, Martin VC, Spreng RN, Szpunar KK. The future of memory: remembering, imagining, and the brain. Neuron. 2012;76:677-694.

10. Gregory WL, Cialdini RB, Carpenter KM. Self-relevant scenarios as mediators of likelihood estimates and compliance: Does imagining make it so? J Pers Soc Psychol. 1982;43:89.

11. Libby LK, Shaeffer EM, Eibach RP, Slemmer JA. Picture yourself at the polls: visual perspective in mental imagery affects self-perception and behavior. Psychol Sci. 2007;18:199-203.

12. Pham LB, Taylor SE. From Thought to Action: Effects of Process-Versus Outcome-Based Mental Simulations on Performance. Personality and Social Psychology Bulletin. 1999;25:250-260.

13. Szpunar KK, Schacter DL. Get real: effects of repeated simulation and emotion on the perceived plausibility of future experiences. J Exp Psychol Gen. 2013;142:323-327.

14. Mathews A, Ridgeway $V$, Holmes EA. Feels like the real thing: imagery is both more 
realistic and emotional than verbal thought. Cogn Emot. 2013;27:217-229.

15. Benoit RG, Szpunar KK, Schacter DL. Ventromedial prefrontal cortex supports affective future simulation by integrating distributed knowledge. Proc Natl Acad Sci U S A. 2014;111:16550-16555.

16. Crane C, Shah D, Barnhofer T, Holmes EA. Suicidal imagery in a previously depressed community sample. Clin Psychol Psychother. 2012;19:57-69.

17. Hales SA, Deeprose C, Goodwin GM, Holmes EA. Cognitions in bipolar affective disorder and unipolar depression: imagining suicide. Bipolar Disord. 2011;13:651-661.

18. Ng, Di Simplicio M, McManus F, Kennerley H, Holmes EA. 'Flash-forwards' and suicidal ideation: A prospective investigation of mental imagery, entrapment and defeat in a cohort from the Hong Kong Mental Morbidity Survey. Psychiatry Research. 2016;246:453-460.

19. Weßlau C, Cloos M, Höfling V, Steil R. Visual mental imagery and symptoms of depression--results from a large-scale web-based study. BMC Psychiatry. 2015;15:308.

20. Holmes EA, Geddes JR, Colom F, Goodwin GM. Mental imagery as an emotional amplifier: application to bipolar disorder. Behav Res Ther. 2008;46:1251-1258.

21. Görgen SM, Joormann J, Hiller W, Witthöft M. The Role of Mental Imagery in Depression: Negative Mental Imagery Induces Strong Implicit and Explicit Affect in Depression. Front Psychiatry. 2015;6:94.

22. O'Donnell C, Di Simplicio M, Brown R, Holmes EA, Burnett Heyes S. The role of mental imagery in mood amplification: An investigation across subclinical features of bipolar disorders. Cortex. 2018;105:104-117.

23. Galton F. Statistics of Mental Imagery. Mind. 1880;5:301-318.

24. Faw B. Conflicting Intuitions May Be Based On Differing Abilities: Evidence from Mental Imaging Research. Journal of Consciousness Studies. 2009;16:45-68.

25. Reisberg D, Pearson DG, Kosslyn SM. Intuitions and introspections about imagery: the role of imagery experience in shaping an investigator's theoretical views. Appl Cogn Psychol. 2003;17:147-160.

26. Morina N, Leibold E, Ehring T. Vividness of general mental imagery is associated with the occurrence of intrusive memories. J Behav Ther Exp Psychiatry. 2013;44:221-226.

27. Debarnot U, Creveaux T, Collet C, Gemignani A, Massarelli R, Doyon J, et al. Sleep-related improvements in motor learning following mental practice. Brain Cogn. 2009;69:398-405.

28. Debarnot U, Castellani E, Valenza G, Sebastiani L, Guillot A. Daytime naps improve motor imagery learning. Cogn Affect Behav Neurosci. 2011;11:541-550.

29. Shearer D, Bruton A, Short S, Roderique-Davies G. Effects of Sleep Quality on Imagery 
Ability in Athletic Populations. Imagin Cogn Pers. 2018;37:394-411.

30. Woud ML, Cwik JC, Blackwell SE, Kleim B, Holmes EA, Adolph D, et al. Does napping enhance the effects of Cognitive Bias Modification-Appraisal training? An experimental study. PLoS One. 2018;13:e0192837.

31. Kleim B, Wysokowsky J, Schmid N, Seifritz E, Rasch B. Effects of Sleep after Experimental Trauma on Intrusive Emotional Memories. Sleep. 2016;39:2125-2132.

32. Porcheret K, lyadurai L, Bonsall MB, Goodwin GM, Beer SA, Darwent M, et al. Sleep and intrusive memories immediately after a traumatic event in emergency department patients. Sleep. 2020. 5 March 2020. https://doi.org/10.1093/sleep/zsaa033.

33. Kusztor A, Raud L, Juel BE, Nilsen AS, Storm JF, Huster RJ. Sleep deprivation differentially affects subcomponents of cognitive control. Sleep. 2019;42.

34. Whitney P, Hinson JM, Nusbaum AT. A dynamic attentional control framework for understanding sleep deprivation effects on cognition. Prog Brain Res. 2019;246:111-126.

35. Palmer CA, Alfano CA. Sleep and emotion regulation: An organizing, integrative review. Sleep Med Rev. 2017;31:6-16.

36. Babson KA, Badour CL, Feldner MT, Bunaciu L. The relationship of sleep quality and PTSD to anxious reactivity from idiographic traumatic event script-driven imagery. J Trauma Stress. 2012;25:503-510.

37. Joiner TE. Why People Die by Suicide. Harvard University Press; 2005.

38. van Bentum JS, Sijbrandij M, Huibers MJH, Huisman A, Arntz A, Holmes EA, et al. Treatment of Intrusive Suicidal Imagery Using Eye Movements. Int J Environ Res Public Health. 2017;14.

39. O'Connor RC, Nock MK. The psychology of suicidal behaviour. Lancet Psychiatry. 2014;1:73-85.

40. Chu C, Buchman-Schmitt JM, Stanley IH, Hom MA, Tucker RP, Hagan CR, et al. The interpersonal theory of suicide: A systematic review and meta-analysis of a decade of cross-national research. Psychol Bull. 2017;143:1313-1345.

41. Dajani DR, Uddin LQ. Demystifying cognitive flexibility: Implications for clinical and developmental neuroscience. Trends Neurosci. 2015;38:571-578.

42. Florentine JB, Crane C. Suicide prevention by limiting access to methods: a review of theory and practice. Soc Sci Med. 2010;70:1626-1632.

43. Miranda R, Gallagher M, Bauchner B, Vaysman R, Marroquín B. Cognitive inflexibility as a prospective predictor of suicidal ideation among young adults with a suicide attempt history. Depression and Anxiety. 2012;29:180-186. 
44. Perrah $\mathrm{M}$, Wichman $\mathrm{H}$. Cognitive rigidity in suicide attempters. Suicide Life Threat Behav. $1987 ; 17: 251-255$.

45. Wenzel A, Beck AT. A cognitive model of suicidal behavior: Theory and treatment. Appl Prev Psychol. 2008;12:189-201.

46. Giegling I, Olgiati P, Hartmann AM, Calati R, Möller H-J, Rujescu D, et al. Personality and attempted suicide. Analysis of anger, aggression and impulsivity. J Psychiatr Res. 2009;43:1262-1271.

47. Gvion Y, Apter A. Aggression, impulsivity, and suicide behavior: a review of the literature. Arch Suicide Res. 2011;15:93-112.

48. Hinson JM, Jameson TL, Whitney P. Impulsive decision making and working memory. J Exp Psychol Learn Mem Cogn. 2003;29:298-306.

49. Green L, Myerson J. A Discounting Framework for Choice With Delayed and Probabilistic Rewards. Psychol Bull. 2004;130:769-792.

50. Mitchell JP, Schirmer J, Ames DL, Gilbert DT. Medial prefrontal cortex predicts intertemporal choice. J Cogn Neurosci. 2011;23:857-866.

51. Mitchell SH. Measures of impulsivity in cigarette smokers and non-smokers. Psychopharmacology. 1999;146:455-464.

52. Baumeister RF. Suicide as escape from self. Psychol Rev. 1990;97:90-113.

53. Gross JJ. Emotion regulation: affective, cognitive, and social consequences. Psychophysiology. 2002;39:281-291.

54. Gross JJ. The Emerging Field of Emotion Regulation: An Integrative Review. Rev Gen Psychol. 1998;2:271-299.

55. Wegner DM. Ironic processes of mental control. Psychol Rev. 1994;101:34-52.

56. Rush AJ, Trivedi MH, Ibrahim HM, Carmody TJ, Arnow B, Klein DN, et al. The 16-Item quick inventory of depressive symptomatology (QIDS), clinician rating (QIDS-C), and selfreport (QIDS-SR): a psychometric evaluation in patients with chronic major depression. Biol Psychiatry. 2003;54:573-583.

57. Altman EG, Hedeker D, Peterson JL, Davis JM. The Altman Self-Rating Mania Scale. Biol Psychiatry. 1997;42:948-955.

58. Buysse DJ, Reynolds CF 3rd, Monk TH, Berman SR, Kupfer DJ. The Pittsburgh Sleep Quality Index: a new instrument for psychiatric practice and research. Psychiatry Res. 1989;28:193-213.

59. Posner K, Brent D, Lucas C, Gould M, Stanley B, Brown G, et al. Columbia-suicide severity rating scale (C-SSRS). New York, NY: Columbia University Medical Center. 2008. 2008. 
60. Wetherall K, Cleare S, Eschle S, Ferguson E, O'Connor DB, O'Carroll RE, et al. From ideation to action: Differentiating between those who think about suicide and those who attempt suicide in a national study of young adults. Journal of Affective Disorders. 2018;241:475-483.

61. Pearson J, Naselaris T, Holmes EA, Kosslyn SM. Mental Imagery: Functional Mechanisms and Clinical Applications. Trends Cogn Sci. 2015;19:590-602.

62. Holmes EA, Arntz A, Smucker MR. Imagery rescripting in cognitive behaviour therapy: images, treatment techniques and outcomes. J Behav Ther Exp Psychiatry. 2007;38:297305.

63. Murphy SE, Clare O'Donoghue M, Drazich EHS, Blackwell SE, Christina Nobre A, Holmes EA. Imagining a brighter future: the effect of positive imagery training on mood, prospective mental imagery and emotional bias in older adults. Psychiatry Res. 2015;230:36-43.

64. Blackwell SE, Browning M, Mathews A, Pictet A, Welch J, Davies J, et al. Positive ImageryBased Cognitive Bias Modification as a Web-Based Treatment Tool for Depressed Adults:

A Randomized Controlled Trial. Clin Psychol Sci. 2015;3:91-111.

65. Robineau F, Meskaldji DE, Koush Y, Rieger SW, Mermoud C, Morgenthaler S, et al. Maintenance of Voluntary Self-regulation Learned through Real-Time fMRI Neurofeedback. Front Hum Neurosci. 2017;11:131.

66. Benoit RG, Davies DJ, Anderson MC. Reducing future fears by suppressing the brain mechanisms underlying episodic simulation. Proc Natl Acad Sci U S A. 2016;113:E8492E8501. 